\title{
Connections from supermassive black holes to white dwarfs
}

\section{Elmar Körding*}

University of Southampton, UK, AIM, CEA Sclay, and Paris Diderot, France

E-mail: Elmar@phys.soton.ac.uk

I present results showing connections between different types of accreting objects. I show the known empirical connections between X-ray binaries and Active Galactic Nuclei, the fundamental plane of black hole activity and the variability plane, as well as highlight some of their applications. In addition, I will argue that also neutron stars and white dwarfs may be included into a single unified scenario of accreting sources.

VII Microquasar Workshop: Microquasars and Beyond

September 1 - 5, 2008

Foca, Izmir, Turkey

${ }^{*}$ Speaker. 


\section{Introduction}

In recent years an increasing number of empirical correlations between different classes of accreting objects have been found. In the study of these connections black hole X-ray binaries (XRBs) play an important role as a 'template' of accretion. The reason for the special position of XRBs in these pictures is that one can well observe the full evolution of an outburst cycle in XRBs with convenient time-scales (months). A similar outburst in active galactic nuclei (AGN) would last millions of years while for cataclysmic variables it lasts only several days. Both cases are therefore hard to observe.

Thus, the definitions of different accretion states used here originate from the study of black hole XRB, which can be found in several distinct states (e.g. [46, 2]). The two main states are the hard state characterised by a hard power-law in the X-ray spectrum and the soft state, where the $\mathrm{X}$-ray spectrum is dominated by multi-color blackbody emission. If a source is in the hard state, one usually observes radio emission which is ascribed to a compact jet [19]. In the soft state no jet is visible and the radio emission is quenched by at least a factor 50. Additionally, there is the intermediate state (IMS) which can be divided into the hard IMS showing an unstable radio jet and the soft IMS where no jet is observed. When the source moves from the hard IMS to the soft IMS one usually observes a radio flare [20]. The accretion state of a compact object is not directly determined by the accretion rate. Only at low accretion rates a $\mathrm{BH}$ is always in a single state: the hard state. Above an accretion rate of $\sim 2 \%$ of the Eddington rate the source can be found any state (hard, soft, IMS). It is not yet known which physical parameter governs the state of a BH. For slightly different definitions (especially for the IMSs) see [37].

Using scaling relations for objects of different black hole mass, it is possible to connect XRBs to AGN and other accreting objects. In addition, one can scale properties found for one class of objects to the other accreting sources and apply them to currently open questions, e.g., the role of jets in AGN feedback. Here, I will present a few of these connections.

\section{The fundamental plane of black hole activity}

A tight radio/X-ray correlation has been found for black hole XRBs [11, 23, 12]. This correlation can be explained assuming that the radio emission originates in a jet [36, 27]. If one simply extends this correlation to AGN one finds that all AGN are too radio loud for a given X-ray luminosity (see Fig. 1). However, if one includes the black hole mass as an additional parameter one obtains a multi-variate correlation between the radio and X-ray luminosities and the black hole masses $[42,18]$. Thus, all accreting black holes populate a plane in this parameter space. An edge-on projection of the correlation is shown in Figure 1.

The correlation can be written as:

$$
\log L_{X} \approx 1.4 \log L_{R}-0.8 M
$$

This non-linear correlation is expected if the radio emission is originating in a scale-invariant conical jet coupled to the accretion disc (e.g., [7, 17]). The X-ray emission can either be explained as inverse-Compton emission from a corona or as synchrotron and synchrotron-self-Compton emission from the jet. Even though the distances of XRBs and AGN are vastly different, it has been shown that the fundamental plane is not a distance artifact [43]. 

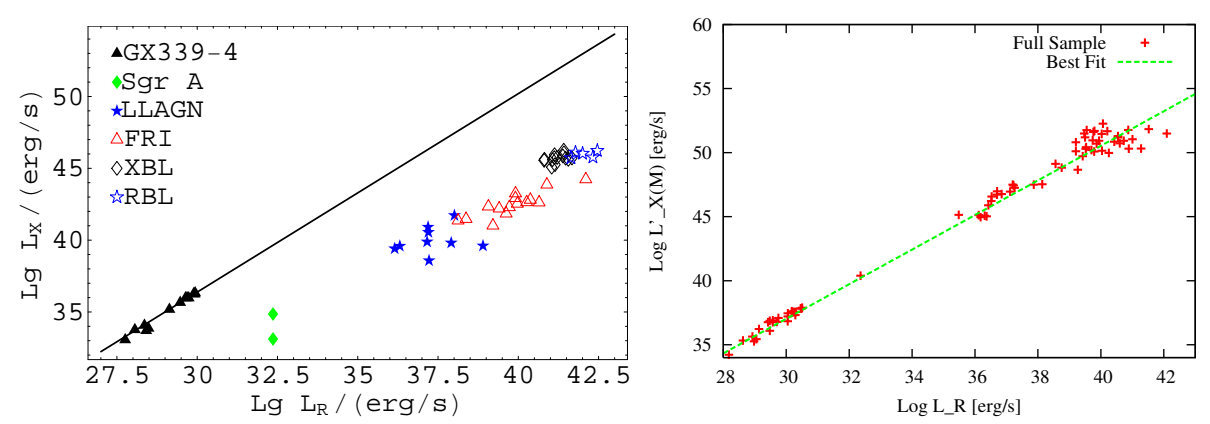

Figure 1: Radio/X-ray correlation for XRBs and AGN. Left panel: effective 'X-ray' emission as a function of the radio luminosity. Besides the radio/X-ray correlation for XRBs one notices that AGN are more radioloud for a given X-ray flux. Right panel: Edge-on projection of the fundamental plane. Plots reproduced from [18, 30] but see also [42] for similar results.

The scatter in correlation and the exact fit values depend on the used sample: If one only uses low-luminosity AGN of LINER type and hard state XRBs the scatter is roughly consistent with the measurement uncertainties [30]. This supports the idea that AGN like XRBs have different states. Low luminosity AGN of LINER type are the best candidates for hard state AGN. If one adds intermediate or soft state objects like Seyferts or FR-I./II Radio galaxies the scatter around the correlation increases.

The interpretations of the fundamental plane suggest that the accretion rate is correlated with the core-radio emission. We [32] investigated this connection further and suggest that the accretion rate can be well estimated from the core-radio emission as

$$
\dot{M}=4 \times 10^{14} \mathrm{~g} \mathrm{~s}^{-1}\left(\frac{L_{8 G H z}}{10^{30} \mathrm{erg} \mathrm{s}^{-1}}\right)^{0.71} .
$$

This relation is established using hard state black hole and neutron star XRBs, which are probably not strongly affected by relativistic beaming. For any application to high power AGN relativistic effects should be taken into account.

Using this accretion rate measure we can rewrite the fundamental plane of black hole activity as:

$$
\frac{L_{X}}{L_{E d d}} \propto\left(\frac{\dot{M}}{\dot{M}_{E d d}}\right)^{2}
$$

Thus, the fundamental plane is simply stating that the X-ray luminosity in units of the Eddington luminosity scales quadratically with the accretion rate in units of Eddington. This is exactly what one expects from inefficient accretion flows.

One can also use the radio luminosity to obtain the total power injected into the jet [26, 34]. This possibility and its applications will be discussed in the next section.

The mass dependency of the fundamental plane can be used to search for intermediate mass black holes. The fundamental plane predicts that the ratio of the radio emission to X-ray emission depends on the black hole mass. It is small for stellar mass black holes but already an intermediatemass black hole of $\sim 10^{4}$ solar masses has a $\sim 400$ times larger radio/X-ray ratio and the supermassive black holes are more than 5 orders of magnitude brighter in the radio bands than a 

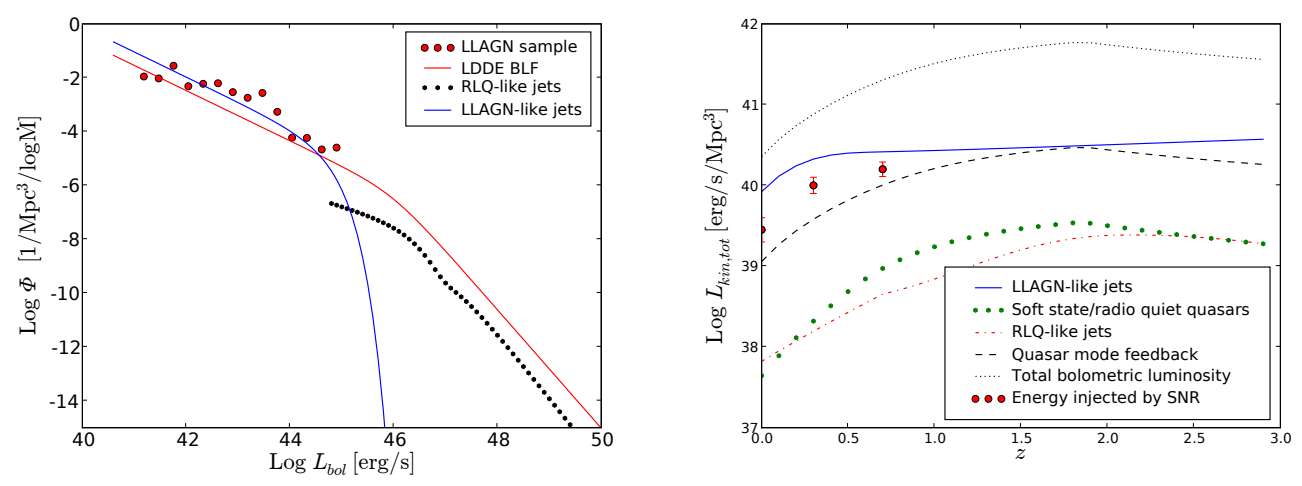

Figure 2: Left panel: Comparison of the accretion rate function derived from radio luminosities with a bolometric luminosity function (BLF) assuming luminosity dependend density evolution (LDDE). Right panel: Total kinetic power injected into the ISM/IGM by jets from AGN of different luminosity. In the local Universe the jets of low luminosity AGN (LLAGN) dominate the total energy input. Only at high redshifts are they comparable to the energy input due to quasars (assuming $5 \%$ efficiency). We also show the kinetic energy injected by radio loud quasars (RLQs).

corresponding XRB. Using this technique [49] found evidence for a $1.8 \times 10^{4}$ solar mass black hole in the globular cluster G1. This result is consistent with measurements based on the velocity curves of the cluster making the suggested black hole one of the strongest candidates for a real intermediate mass black hole.

\section{Accretion rate functions and jet powers}

As presented in the previous section accretion rates as well as jet powers can be estimated using the unbeamed core radio fluxes. These estimators are not limited to XRBs but they are also applicable to AGN if one accounts for relativistic beaming effects. Using radio luminosity functions one can therefore construct accretion rate functions (ARFs) for radio loud objects as well as kinetic luminosity functions [26, 34]. At low luminosity this is directly possible as low luminosity radio jets do not seem to be strongly beamed [10]. At high radio luminosities, i.e., for FR-II radio galaxies, the core radio flux is likely to be strongly beamed. However, low frequency radio emission coming from the lobes of the object can then be used to estimate the jet power and thus the accretion rate [47, 34].

The two contributions of low power 'LLAGN-like' jets and those of high power jets need to be treated differently. In Fig. 2 we show the local bolometric luminosity function of AGN by Hopkins et al. [29] as well as our accretion rate functions. The ARFs derived from LLAGN are based on radio luminosity functions of $15 \mathrm{GHz}$ VLA observations to ensure that the observed radio luminosity originates from the core of the jet [45]. In addition we also show the ARF for high power objects (i.e., FR-II Radio galaxies, radio loud quasars ) derived from $158 \mathrm{MHz}$ radio luminosities. The difference of these different luminosity functions describes the fraction of 'radioloud' objects. At low luminosities the LLAGN ARF is of a similar magnitude or even above the bolometric luminosity function of AGN. At high luminosities, there are significantly less radio loud 

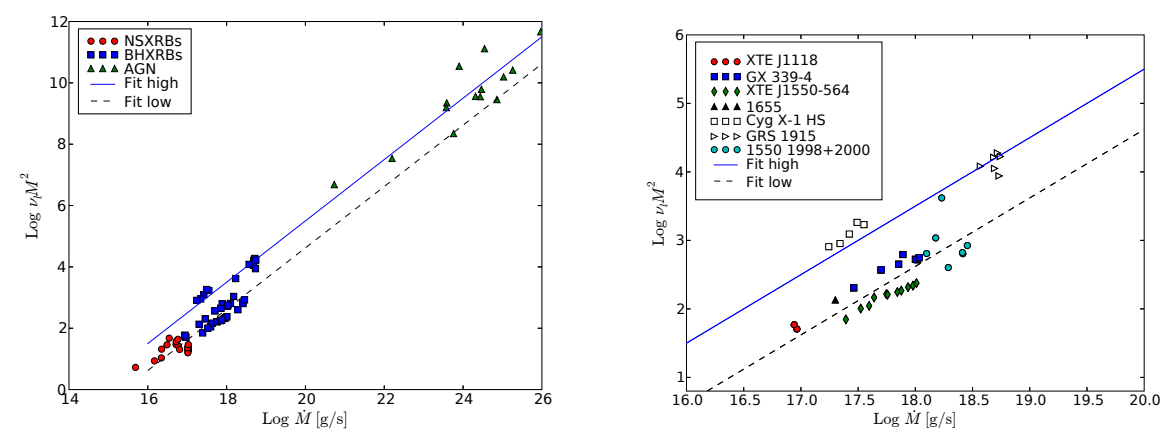

Figure 3: Edge-on projection of the variability plane for AGN and hard and soft state XRB. While the left panel shows the full correlation the right panel zooms in on the XRBs. The linear dependence on the accretion rate is seen both in AGN alone as well as in hard and soft state XRBs.

objects than normal quasars. For details of the derivation of the ARF and the results see [34] or as an alternative approach $[26,41]$.

As we are estimating the accretion rate from jet properties, we can also derive the total kinetic power injected into the inter-galactic and inter stellar medium (IGM/ISM) by AGN jets. This relation is normalised using a sample of measured kinetic jet powers derived from jet inflated Xray cavities [1]. The total power injected by the different classes of AGN is shown in Fig. 2. In the local universe the total kinetic power injected into the ISM/IGM is dominated by low luminosity AGN and not by the rare radio loud quasars. Only at high redshifts is the so called 'quasar mode' feedback of similar value to the total power as LLAGN. In the quasar mode one assumes that $\sim 5 \%$ of the energy produced by the quasar is available for feedback ([15], the exact feedback mechanism is not yet fully established). Overall, the total kinetic power injected into the IGM by AGN is similar to that injected by supernovae.

\section{The variability plane}

It has been long recognised that the power spectral density (PSD) of the X-ray light-curves of AGN are similar to those of black hole XRBs [38], if one scales the observed frequencies linearly with the black hole mass. Both, the PSD of AGN as well as those of a soft state XRB can be described by a broken power law. At low Fourier frequencies the PSD can be described as a power law with index $\sim-1$ which steepens around the characteristic timescale $\left(v_{h b}\right)$ to a power law with index $\sim-2[25,14,16]$. Uttley et al. [50] noted that the correlation between the characteristic timescales and the black hole mass has significant scatter and there seems to be an indication that the bolometric luminosity influences the characteristic frequencies as well. Also for XRBs it has been shown that the variability timescales depend on the accretion rate [52] or on their radio luminosities [44].

Thus, it is likely that also the characteristic timescales do not only depend on the black hole mass but also on the accretion rate. Like the 'fundamental plane of black hole activity' all accreting objects may populate a plane in the space given by the black hole mass, accretion rate and the 
characteristic timescales. And indeed, [39] report the existence of such a plane. In Fig. 3 I show an edge-on projection of the variability plane of soft state objects in the left panel. We find:

$$
v_{h b} \propto \frac{\dot{M}}{M^{2}} \quad \text { or } \quad M v_{h b} \propto \frac{\dot{M}}{M}
$$

The characteristic variability frequency in units of the light-crossing time of the central object depends linearly on the accretion rate in Eddington units, and is therefore scale invariant in respect to the black hole mass. In case that the X-ray emission originates from the accretion flow, the correlation suggests that the accretion flow is - at least approximately - scale invariant.

The PSD of hard state X-ray binaries shows more features than those of a soft state object. It is usually fitted by a number of Lorentzians [3]. We argue in [35] that one can identify the characteristic timescale found in AGN and soft state XRBs with the characteristic frequency $v_{l}$ of the lower high frequency Lorentzian. In Fig. 3 a sample of hard state XRBs is shown. To better visualise the behaviour in stellar object the right panel shows only stellar black holes. There seems to be a constant offset between hard state objects and soft state objects. We also show two outbursts of XTE J1550 (1998 and 2000), where the source seem to be in a transition state. It starts off at the hard state scaling and moves towards the soft state scaling. Thus, the frequencies do not only depend on the accretion rates and black hole masses but also on a parameter governing the accretion state of the object.

Besides black holes, one can also add neutron stars with measured frequencies $v_{l}$ of the noise components described by the lower high frequency Lorentzian.

One application of this plane is the estimation of the black hole masses in ultra-luminous $\mathrm{X}$ ray sources. Casella et al. [9] used the variability plane to estimate the mass of M82 X-1 and NGC 5408 X-1. They conclude that both sources contain black holes of masses between 100 and 1300 solar masses. While this application is a promising step towards measuring black hole masses using variability time-scales, the systematic and statistical uncertainties of the fundamental plane for mass measuring are still very large and only provide an additional argument for the intermediate mass of the object.

\section{Quasi-periodic oscillations}

Quasi-periodic oscillations are a common phenomenon of neutron star and black hole XRBs [51]. They are well known to be correlated with spectral variations as well as with the luminosity of the source. One can distinguish three main QPOs types: type A, B and C [8]. The classification depends on the width of the QPO feature as well as on their relative strength. A similar phenomenon is observable for white dwarfs (e.g., [54]). As these features are so prominent in XRBs they have been long searched in AGN. However, while there are some claims of AGN QPOs there had been until recently - no significant detection of a QPO [53]. Only recently Gierlinski et al. [24] reported the first likely detection of a QPO (see fig. 4). The QPO frequency is in agreement with the value expected from scaling linearly with black hole mass from XRBs. However, it is not yet clear to which QPO type this QPO belongs. 


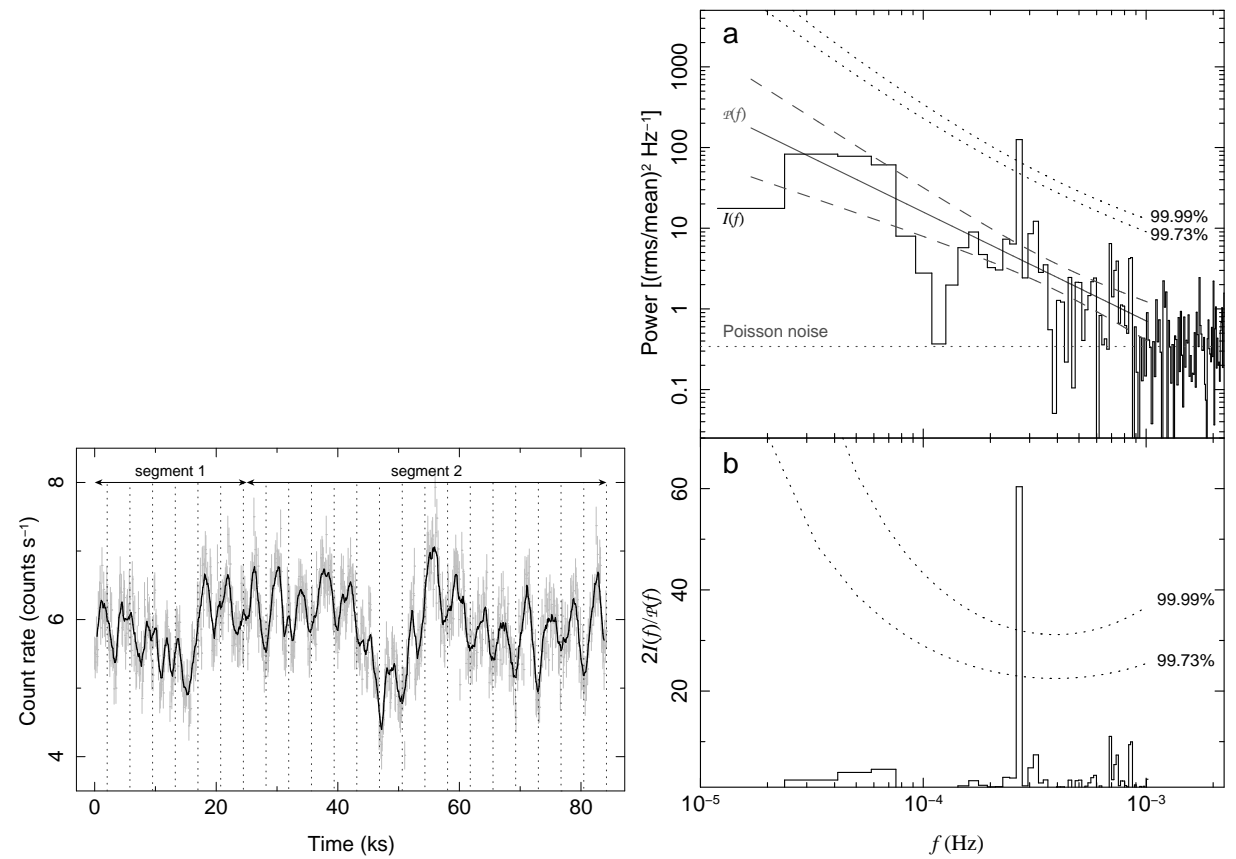

Figure 4: Quasi-periodic oscillations in an AGN: The left panel shows the light-curve together with an indication of the periodicity. The right panel presents the power spectral density and the significance of any peaks. Plots reproduced with permission from [24]

\section{AGN states}

The aforementioned similarities between XRBs and AGN suggest that also AGN do have accretion states. As argued, the fundamental plane of black hole activity suggests that low luminosity AGN may be the analogue of hard state XRBs. That had already been proposed by [28]. Also other classes of the AGN zoo had been associated with different XRB states (e.g., [40]).

To proceed further it has been suggested to generalise tools to study accretion states developed for XRBs for all accreting objects. To identify accretion states in XRBs one usually uses hardnessintensity diagrams (HIDs) to identify accretion states and their association to jets (but note that especially during the intermediate states timing information is needed for a reliable state identification). In such a diagram one plots X-ray counts (intensity) against the X-ray hardness (e.g, counts in a hard band divided by counts in a soft band). A sketch of such a diagram is shown in Fig. 5. Jet launching hard state objects are found on the right side of the diagram while soft state objects are found on the left side. During the transition from a hard to a soft state, the source first enters the hard IMS - associated with an increasingly unstable jet, which is quenched once the source moves to the soft IMS crossing the so called "jet line" [20].

As an important check of XRB-AGN unification, we consider whether we can identify accretion states in an equivalent diagram for AGN. One of the reasons why HIDs work for XRBs is that both the power-law contribution (due to Comptonization or jet emission) and the disc emission is found in the X-ray band. The hardness measure compares the blackbody flux to the power law flux. However, for AGN the disc contribution is in the optical or the UV. We therefore have to generalise 


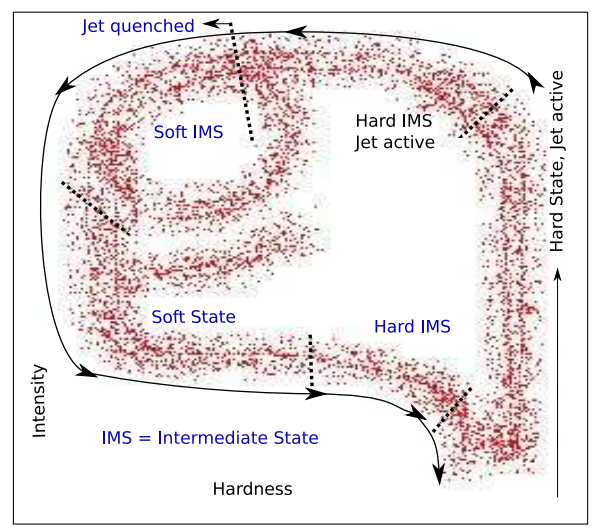

Figure 5: Sketch of an hardness-intensity diagram. On the X-axis we plot the X-ray color while the Y-axis corresponds to the total measured counts $(\propto$ flux).

the hardness measure to the non-thermal fraction, a ratio of the power law flux to the total flux:

$$
f=\frac{L_{P L}}{L_{P L}+L_{D}}
$$

where $L_{D}$ is the disc luminosity and $L_{P L}$ is the power law luminosity. For XRBs both quantities can be measured in the X-rays. For AGN the power law luminosity is still measurable in the X-ray band, but the disc emits in the optical or UV. We will refer to plots showing the total luminosity $\left(L_{D}+L_{P L}\right)$ as a function of the non-thermal fraction as disc-fraction luminosity diagrams (DFLD).

To study the dependency of the jet emission on the position DFLD we crosscorrelate the quasars of the Sloan Digital Sky Survey (SDSS, data release 5) with the ROSAT all sky survey and the FIRST radio catalogue. For each source detected in the SDSS and by ROSAT we can calculate the non-thermal fraction. As a measure of the jet activity we use the radio-loudness parameter $R$ defined as the ratio of the radio flux to the B-band flux. To extend the DFLD to lower luminosities we also include a sample of low-luminosity AGN with radio measurements from [45].

In the left panel of Fig. 6 we show a contour map of the radio loudness $R$ as a function of the position in the DFLD. We have divided the diagram into $10 \times 20$ bins. For each bin we calculate the average $R$ if there are more than 10 SDSS quasars in the bin.

The top part of the diagram is due to the SDSS quasars while the lower part is due to the low-luminosity AGN. In the top part, sources on the lower left are significantly less radio loud than those on the upper right. At lower luminosities the sources mainly populate the right side of the diagram and they are more radio loud than the quasars (as already shown by [28]). In [33] we discuss in detail why we believe that the described effect is real and not an artifact of the detection limits or the sample selection. We also discuss beaming effects and the effect of the BH mass and conclude that they are unlikely to create the observed distribution.

On the right side of Fig. 6 we present a DFLD simulated for 100 outbursts of XRBs. During each outburst the source moves from the initial hard state up in luminosity until it makes a transition via the hard jet emitting IMS to the soft IMS and the soft state. Here, it decreases again in flux until it makes a transition back to the hard state. To obtain the radio loudness we assumed that the XRB follows the radio/X-ray correlation during the hard state. In the hard IMS we assumed a similar 
radio luminosity as in the preceding hard state, which is quenched by a factor 100 as the source moves to the soft IMS. The simulated DFLD is similar to the measured DFLD. This suggests that AGN have the same accretion states as XRBs with associated jet properties.
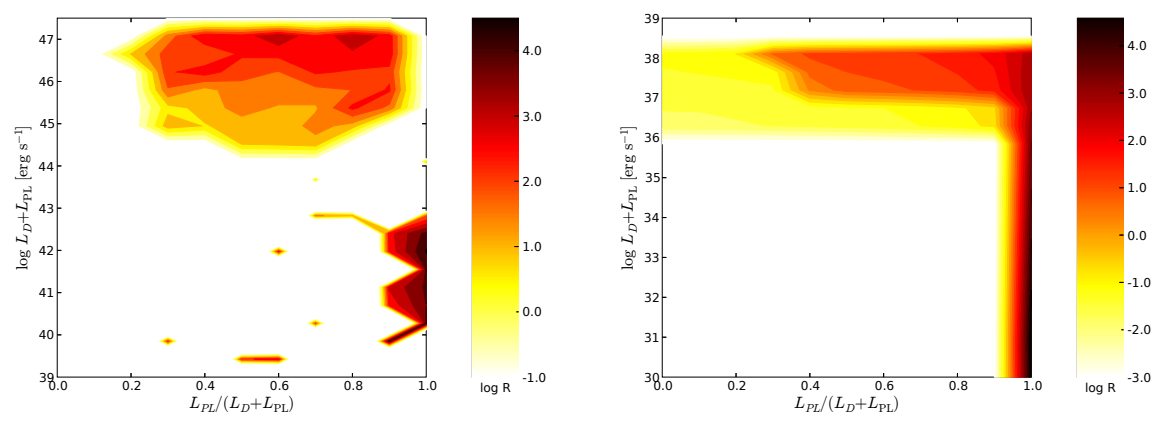

Figure 6: Average radio loudness as a function of the position on the DFLD. Left panel: SDSS sample together with a low-luminosity sample. Right panel: Monte Carlo simulation of 100 XRB outbursts.

\section{Cataclysmic Variables}

Jets are also found in accreting white dwarf systems. For example, the symbiotic star CH Cyg shows strong radio jets that can be directly imaged [13]. Also this jet seems to be coupled to the accretion disc. For a jet ejection event the source first needs to show a dip in the optical light-curve created by the disk [48]. This is similar to the behaviour seen in GRS1915+105

Cataclysmic variables (CVs) show violent outbursts similar to XRBs. In fact, one assumes that outbursts in both classes of objects are triggered by the same instability first explored for the white dwarfs (dwarf novae instability see e.g., [21]). The similarity between XRBs and CVs is most prominent for dwarf novae. These non-magnetic, weakly accreting white dwarfs show fairly regular outbursts that are thought to be powered only by accretion. Thus, they are a different class of objects than normal novae, where the initial injection of energy is due to thermonuclear burning. Recently, it has been found that CVs during outburst show highly variable radio emission interpreted as jet emission. As the radio emission is, even for the nearest CVs, very weak and therefore nearly unexplored.

General relativity does not play an important role in white dwarfs, nevertheless, they do show jets. Thus, one of the key questions that can be answered by studying CVs and comparing them to the relativistic black hole $\mathrm{XRBs}$, is to what extend general relativity affects the jet launching processes.

The analogy between XRBs and CVs can be visualised using a disc-fraction luminosity diagram (the generalised version of the HID see above and figure 7). Such a diagram for a CV is shown in comparison with a black hole XRB and a neutron star XRB. For all three classes the luminosity of the outburst is first dominated by the X-ray power law component (originating from optically thin components) until a transition switches the source to a disc/boundary layer dominated state. In all cases one observes a hysteresis effect. 

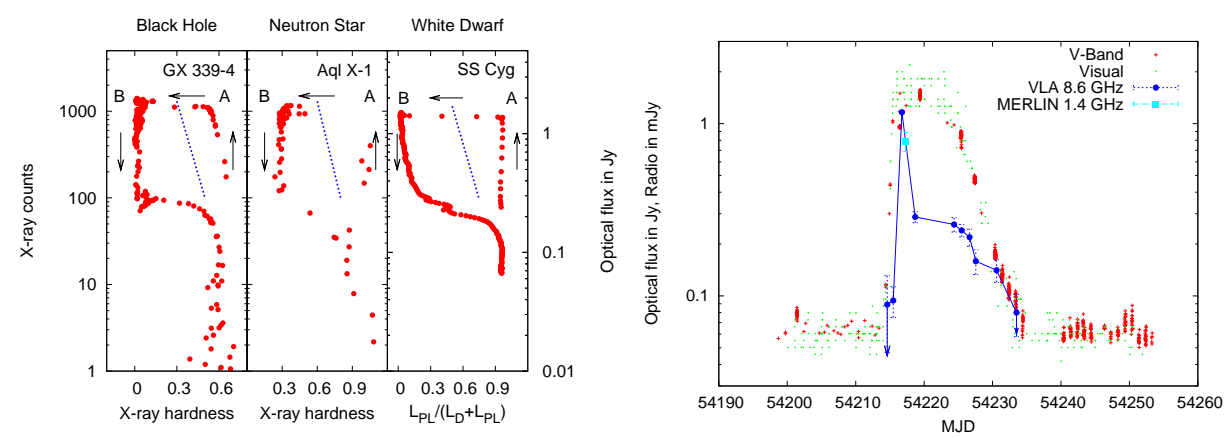

Figure 7: Left panel: Generalised hardness intensity diagram for a black hole, a neutron star and a white dwarf. Right panel: Optical light-curve of the dwarf nova SS Cyg in comparison with its radio flux.

The similarity of the 'HID' suggests that also the jet emission might be similar in CVs. Radio emission, which is often used as a tracer for jets, from CVs has been reported for a while [4, 6], but was usually not been reproducible [22]. It has thus been suggested that the radio emission is correlated with the optical outburst [5]. This has been confirmed by [31] with a detailed radio lightcurve of SS Cyg. Near the onset of the outburst one observes a radio flare ( $1 \mathrm{mJy}$ ) that declines rapidly to fairly steady plateau emission $(0.3 \mathrm{mJy})$. The latter emission declines slower than the optical light-curve of the outburst, while the radio spectrum is flat to inverted. This behaviour has been reproduced with a second outburst. The outburst cycle in the radio bands is similar to those seen in XRBs just on much shorter timescales (days compared to weeks to months): At the beginning of the XRB outburst one observes strong radio emission in the hard state, a flare during the transition to the soft state followed by weaker (neutron stars) or quenched radio emission in the soft state.

The radio luminosity of the $\mathrm{CV}$ is in agreement with extrapolations of the radio luminosities observed in XRBs, if one accounts for the different accretion rates and the larger radius of the central object (which reduces the total power liberated in the accretion flow). These similarities in combination with the high-brightness temperature and variability of the emission led [31] to conclude that the emission is most likely synchrotron emission that is best explained as originating in a jet.

\section{Conclusions}

We now have several features that can be scaled from XRBs to AGN and even to other objects using the black hole mass and the accretion rate as their main parameters. Both the fundamental plane as well as the variability plane can be interpreted in such a way: for the fundamental plane one observes $\mathrm{X}$-ray luminosity as a function of the black hole mass and the accretion rate; for the variability plane its the break frequency.

EK thanks Rob Fender, Tomaso Belloni, Robert Dunn, Christian Knigge, Stephane Corbel, Tom Maccarone and Sebastian Jester for helpful discussions. 


\section{References}

[1] S. W. Allen, R. J. H. Dunn, A. C. Fabian, G. B. Taylor, and C. S. Reynolds, The relation between accretion rate and jet power in X-ray luminous elliptical galaxies, MNRAS 372, 21-30 (2006) [astro-ph/0602549].

[2] T. Belloni, J. Homan, P. Casella, M. van der Klis, E. Nespoli, W. H. G. Lewin, J. M. Miller, and M. Méndez, The evolution of the timing properties of the black-hole transient GX 339-4 during its 2002/2003 outburst, A\&A 440, 207-222 (2005).

[3] T. Belloni, D. Psaltis, and M. van der Klis, A Unified Description of the Timing Features of Accreting $X$-Ray Binaries, ApJ 572, 392-406 (2002).

[4] A. O. Benz, E. Fuerst, and A. L. Kiplinger, First detection of radio emission from a dwarf nova, Nature 302, 45-+ (1983).

[5] A. O. Benz, M. Gudel, and J. A. Mattei, Radio Emission of Dwarf Novae in proceedings of Radio Emission from the Stars and the Sun, (A. R. Taylor and J. M. Paredes, eds.), Astronomical Society of the Pacific Conference Series, vol. 93, 1996, pp. 188-+.

[6] A. O. Benz and M. Guedel, VLA detection of radio emission from a dwarf nova, A\&A 218, 137-140 (1989).

[7] R. D. Blandford and A. Königl, Relativistic jets as compact radio sources, ApJ 232, 34-48 (1979).

[8] P. Casella, T. Belloni, and L. Stella, The ABC of Low-Frequency Quasi-periodic Oscillations in Black Hole Candidates: Analogies with Z Sources, ApJ 629, 403-407 (2005) [arXiv: astro-ph/0504318].

[9] P. Casella, G. Ponti, A. Patruno, T. Belloni, G. Miniutti, and L. Zampieri, Weighing the black holes in ultraluminous X-ray sources through timing, MNRAS 387, 1707-1711 (2008) [arXiv:0804.3378].

[10] M. H. Cohen, M. L. Lister, D. C. Homan, M. Kadler, K. I. Kellermann, Y. Y. Kovalev, and R. C. Vermeulen, Relativistic Beaming and the Intrinsic Properties of Extragalactic Radio Jets, ApJ 658, 232-244 (2007) [arXiv: astro-ph/0611642].

[11] S. Corbel, R. P. Fender, A. K. Tzioumis, M. Nowak, V. McIntyre, P. Durouchoux, and R. Sood, Coupling of the $X$-ray and radio emission in the black hole candidate and compact jet source GX 339-4, A\&A 359, 251-268 (2000).

[12] S. Corbel, E. Koerding, and P. Kaaret, Revisiting the radio/X-ray flux correlation in the black hole V404 Cyg: from outburst to quiescence, MNRAS 389, 1697-1702 (2008).

[13] M. M. Crocker, R. J. Davis, R. E. Spencer, S. P. S. Eyres, M. F. Bode, and A. Skopal, The symbiotic star CH Cygni - III. A precessing radio jet, MNRAS 335, 1100-1108 (2002) [arXiv:astro-ph/0209097].

[14] W. Cui, S. N. Zhang, W. Focke, and J. H. Swank, Temporal Properties of Cygnus X-1 during the Spectral Transitions, ApJ 484, 383-(1997).

[15] T. Di Matteo, V. Springel, and L. Hernquist, Energy input from quasars regulates the growth and activity of black holes and their host galaxies, Nature 433, 604-607 (2005) [a st ro-ph/0 02199 ].

[16] R. Edelson and K. Nandra, A Cutoff in the X-Ray Fluctuation Power Density Spectrum of the Seyfert 1 Galaxy NGC 3516, ApJ 514, 682-690 (1999). 
[17] H. Falcke and P. L. Biermann, The jet-disk symbiosis. I. Radio to X-ray emission models for quasars., A\&A 293, 665-682 (1995).

[18] H. Falcke, E. Körding, and S. Markoff, A scheme to unify low-power accreting black holes. Jet-dominated accretion flows and the radio/X-ray correlation, A\&A 414, 895-903 (2004).

[19] R. P. Fender, Powerful jets from black hole X-ray binaries in low/hard X-ray states, MNRAS 322, 31-42 (2001).

[20] R. P. Fender, T. M. Belloni, and E. Gallo, Towards a unified model for black hole X-ray binary jets, MNRAS 355, 1105-1118(2004).

[21] J. Frank, A. King, and D. J. Raine, Accretion Power in Astrophysics: Third Edition, Accretion Power in Astrophysics, by Juhan Frank and Andrew King and Derek Raine, pp. 398. ISBN 0521620538. Cambridge, UK: Cambridge University Press, February 2002., February 2002.

[22] E. Fuerst, A. Benz, W. Hirth, M. Geffert, and A. Kiplinger, Radio emission of cataclysmic variable stars, A\&A 154, 377-+ (1986).

[23] E. Gallo, R. P. Fender, and G. G. Pooley, A universal radio-X-ray correlation in low/hard state black hole binaries, MNRAS 344, 60-72 (2003).

[24] M. Gierliński, M. Middleton, M. Ward, and C. Done, A periodicity of $\sim$ lhour in X-ray emission from the active galaxy RE J1034+396, Nature 455, 369-371 (2008).

[25] A. R. Green, I. M. McHardy, and H. J. Lehto, On the nature of rapid X-ray variability in active galactic nuclei, MNRAS 265, 664-(1993).

[26] S. Heinz, A. Merloni, and J. Schwab, The Kinetic Luminosity Function and the Jet Production Efficiency of Growing Black Holes, ApJ 658, L9-L12 (2007) [astro-ph/0 02211 ].

[27] S. Heinz and R. A. Sunyaev, The non-linear dependence of flux on black hole mass and accretion rate in core-dominated jets, MNRAS 343, L59-L64 (2003).

[28] L. C. Ho, “Low-State” Black Hole Accretion in Nearby Galaxies, Ap\&SS 300, 219-225 (2005).

[29] P. F. Hopkins, G. T. Richards, and L. Hernquist, An Observational Determination of the Bolometric Quasar Luminosity Function, ApJ 654, 731-753 (2007) [astro-ph/0605678].

[30] E. Körding, H. Falcke, and S. Corbel, Refining the fundamental plane of accreting black holes, A\&A 456, 439-450 (2006) [astro-ph/0603117].

[31] E. Körding, M. Rupen, C. Knigge, R. Fender, V. Dhawan, M. Templeton, and T. Muxlow, A Transient Radio Jet in an Erupting Dwarf Nova, Science 320, 1318-(2008) [0 806.1002 ].

[32] E. G. Körding, R. P. Fender, and S. Migliari, Jet-dominated advective systems: radio and X-ray luminosity dependence on the accretion rate, MNRAS 369, 1451-1458 (2006).

[33] E. G. Körding, S. Jester, and R. Fender, Accretion states and radio loudness in active galactic nuclei: analogies with X-ray binaries, MNRAS 372, 1366-1378 (2006) [astro-ph / 0608628 ].

[34] E. G. Körding, S. Jester, and R. Fender, Measuring the accretion rate and kinetic luminosity functions of supermassive black holes, MNRAS, 1100-(2007) [arXiv:0710 .1718].

[35] E. G. Körding, S. Migliari, R. Fender, T. Belloni, C. Knigge, and I. McHardy, The variability plane of accreting compact objects, MNRAS 380, 301-310 (2007) [arXiv: 0706.2959 ].

[36] S. Markoff, M. Nowak, S. Corbel, R. Fender, and H. Falcke, Exploring the role of jets in the radio/X-ray correlations of GX 339-4, A\&A 397, 645-658 (2003). 
[37] J. McClintock and R. Remillard, in "Compact Stellar X-ray Sources," eds. W.H.G. Lewin and M. v an der Klis, Cambridge University Press (2006).

[38] I. McHardy, EXOSAT observations of variability in active galactic nuclei, Memorie della Societa Astronomica Italiana 59, 239-259 (1988).

[39] I. M. McHardy, E. Koerding, C. Knigge, P. Uttley, and R. P. Fender, Active galactic nuclei as scaled-up Galactic black holes, Nature 444, 730-732 (2006) [astro-ph/ 0612273 ].

[40] D. L. Meier, The Association of Jet Production with Geometrically Thick Accretion Flows and Black Hole Rotation, ApJ 548, L9-L12 (2001).

[41] A. Merloni and S. Heinz, A synthesis model for AGN evolution: supermassive black holes growth and feedback modes, MNRAS 388, 1011-1030 (2008) [0805.2499].

[42] A. Merloni, S. Heinz, and T. Di Matteo, A Fundamental Plane of black hole activity, MNRAS 345, 1057-1076 (2003).

[43] A. Merloni, E. Körding, S. Heinz, S. Markoff, T. Di Matteo, and H. Falcke, Why the fundamental plane of black hole activity is not simply a distance driven artifact, New Astronomy 11, 567-576 (2006) [astro-ph/0601286].

[44] S. Migliari, R. P. Fender, and M. van der Klis, Correlation between radio luminosity and X-ray timing frequencies in neutron star and black hole X-ray binaries, MNRAS, 799-(2005).

[45] N. M. Nagar, H. Falcke, and A. S. Wilson, Radio sources in low-luminosity active galactic nuclei. IV. Radio luminosity function, importance of jet power, and radio properties of the complete Palomar sample, A\&A 435, 521-543 (2005).

[46] M. A. Nowak, Toward a Unified View of Black-Hole High-Energy States, PASP 107, 1207+ (1995).

[47] S. Rawlings and R. Saunders, Evidence for a common central-engine mechanism in all extragalactic radio sources, Nature 349, 138-140 (1991).

[48] J. L. Sokoloski and S. J. Kenyon, CH Cygni. I. Observational Evidence for a Disk-Jet Connection, ApJ 584, 1021-1026 (2003) [astro-ph/0211040].

[49] J. S. Ulvestad, J. E. Greene, and L. C. Ho, Radio Emission from the Intermediate-Mass Black Hole in the Globular Cluster G1, ApJ 661, L151-L154 (2007) [arXiv: 0704.1458 ].

[50] P. Uttley and I. M. McHardy, X-ray variability of NGC 3227 and 5506 and the nature of active galactic nucleus 'states', MNRAS, 818-(2005).

[51] M. van der Klis, Quasi-periodic oscillations and noise in low-mass X-ray binaries, ARA\&A 27, 517-553 (1989).

[52] M. van der Klis, A Possible Explanation for the "Parallel Tracks" Phenomenon in Low-Mass X-Ray Binaries, ApJ 561, 943-949 (2001).

[53] S. Vaughan and P. Uttley, Detecting X-ray QPOs in active galaxies, Advances in Space Research 38 , 1405-1408 (2006) [arXiv:astro-ph/0 506456 ].

[54] B. Warner, P. A. Woudt, and M. L. Pretorius, Dwarf nova oscillations and quasi-periodic oscillations in cataclysmic variables - III. A new kind of dwarf nova oscillation, and further examples of the similarities to X-ray binaries, MNRAS 344, 1193-1209 (2003) [astro-ph/0306085]. 